\title{
The role of PoCUS in the assessment of COVID-19 patients
}

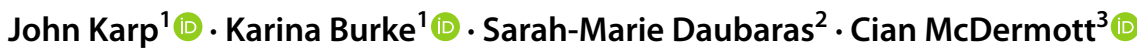

Received: 3 November 2020 / Accepted: 8 April 2021 / Published online: 19 April 2021

(c) The Author(s) 2021

\begin{abstract}
The Coronavirus disease 19 (COVID-19) pandemic has increased the burden of stress on the global healthcare system in 2020. Point of care ultrasound (PoCUS) is used effectively in the management of pulmonary, cardiac and vascular pathologies. POCUS is the use of traditional ultrasound imaging techniques in a focused binary manner to answer a specific set of clinical questions. This is an imaging technique that delivers no radiation, is inexpensive, ultraportable and provides results instantaneously to the physician operator at the bedside. In regard to the pandemic, PoCUS has played a significant adjunctive role in the diagnosis and management of co-morbidities associated with COVID-19. PoCUS also offers an alternative method to image obstetric patients and the pediatric population safely in accordance with the ALARA principle. Finally, there have been numerous PoCUS protocols describing the effective use of this technology during the COVID-19 pandemic.
\end{abstract}

Keywords PoCUS · Ultrasound · COVID-19 · Coronavirus

\section{Introduction}

COVID-19 is a novel virus from the coronavirus family. It has a mean incubation period of 4-5 days however, $[1,2]$ COVID-19 spreads via respiratory droplets from person-toperson and causes an acute respiratory illness [3]. While the information on the pathogenesis and pathology of the disease is quickly evolving and vast with large variations on the severity of disease, the major symptoms include fever, fatigue, dry cough, dyspnea and anosmia [4]. Since notification of the first instances of a cluster of pneumonias with an unknown cause in Wuhan, China on the 31st of December 2019, COVID-19 has spread quickly throughout 216

John Karp

johnkarp@rcsi.com

Karina Burke

karinaburke@rcsi.com

Sarah-Marie Daubaras

sarahdaubaras@rcsi.com

Cian McDermott

cianmcdermott@gmail.com

1 School of Medicine, Royal College of Surgeons in Ireland, Dublin, Ireland

2 Emergency Department, St James Hospital, Dublin, Ireland

3 Emergency Department and Emergency Ultrasound Education, Mater University Hospital, Dublin, Ireland countries globally [4]. Declared a pandemic by the WHO on March 11th, 2020, the disease has led to 35 million infections and 1 million deaths [5]. The rise of COVID-19 globally has placed significant pressure on healthcare and its resources.

The current gold standard diagnostic method is reverse transcription-PCR (RT-PCR) testing of a nasopharyngeal swab [6, 7]. Alternatively, chest X-ray (CXR) and chest computed tomography (CT) are commonly used imaging modalities. A CXR is less sensitive than a chest CT and they both subject the patient to varying degrees of radiation $[8,9]$. PoCUS refers to the act of using ultrasound during a standard clinical assessment as an adjuvant diagnostic tool. Ultrasonography has proven to be an alternative solution in offering a safe and quick first-line bedside diagnostic exam for COVID-19 lung, cardiac and venous thromboembolic manifestations [10-12]. In this review, the implications of PoCUS in the management of COVID-19 patients will be explored.

\section{Methods}

To complete this literary review, articles from PubMed.org database were reviewed for eligibility. No language, date or age restrictions were applied. A search string was designed using the following terms: COVID-19, coronavirus, point 
of care ultrasound, and PoCUS with the following filters applied: clinical trial, meta-analysis, randomized controlled trial, review, systematic review. Papers deemed eligible based on abstract review were accessed as full-length articles for analysis.

\section{PoCUS advantages and limitations}

Nanotechnological advancements in technology have enabled ultrasound to become an affordable and portable imaging modality. Whether it is a cart-based ultrasound machine or a handheld device, a scan can be conducted at the bedside quickly and affordably while providing physicians with results in real time [13, 14]. In regard to COVID-19, PoCUS offers a non-invasive option to screen and trend patients safely. Disinfection protocols include sheathing and disinfection of the ultrasound transducer [10]. Although an ultrasound device may act as a fomite for COVID-19, following a simple checklist can assist physicians in the disinfection process to safely reduce contamination (Table 1) [11, 15-18].

Additionally, the portability of PoCUS reduces the risk of transmission to staff and equipment exposure, which in turn conserves valuable personal protective equipment (PPE) and supplies [11]. Rather than transporting a patient through the hospital to the radiology department, a dedicated ultrasound machine in a COVID-19 unit may be brought to the patient's bedside. A single physician can interact with the patient minimizing staff exposure while performing an exam.

In comparison to CT imaging, PoCUS is a non-ionizing imaging modality which offers comparable sensitivity and accuracy in the diagnosis of COVID-19 lung involvement [19]. In comparison to a portable X-ray which correlated to CT lung findings by $62 \%$, PoCUS paralleled CT lung findings of COVID-19 by $87 \%$ [20]. Another study concluded that while comparing ultrasound to CT images of alveolarinterstitial patterns in the same COVID-19 patients, PoCUS had a sensitivity of $60 \%$ compared to only $39 \%$ in CT images [21]. Additionally, PoCUS is extremely beneficial in monitoring the progression of cardiac and venous abnormalities associated with COVID-19 [11].

Although PoCUS is a useful tool, it does carry limitations. To acquire clinically useful images, the operator must have sufficient experience using an ultrasound machine [11, 13]. Patients with an elevated body habitus or compromised status may impair the operator's ability to obtain a clear visual [22]. In addition, PoCUS images may be difficult to interpret, which may lead to missed findings or require further investigations using alternative imaging modalities [11].

\section{PoCUS for COVID-19 lung involvement}

The organ system most commonly involved by the coronavirus is the respiratory tract. It can manifest as a mild viral pneumonia or acute respiratory distress syndrome [23, 24]. Lung ultrasound (LUS) has proven to be an invaluable firstline diagnostic tool when it comes to COVID-19 patients. It has an overall diagnostic sensitivity and specificity of $90.2 \%$ (67.6-99.3\%) and 88.8\% (81.8-94.4\%) in COVID-19 pneumonia when compared to CXR, CT and clinical exam [25]. Additionally, while COVID-19 has a preference to affect the posterior-basal lung zones, ultrasound provides effective visualization of the lung's peripheries [10, 26-30]. LUS is not capable of visualising central lung lesions-these are best seen on cross-sectional CT imaging.

Common pathologies visualized using LUS during COVID-19 are multifocal B lines, sub-pleural/lobar consolidations with decreased doppler signal, bilateral alveolarinterstitial pattern, pleural irregularities, air bronchograms and less commonly pleural effusions [9, 11, 18, 30-33]. B-lines are defined as reverberation artifacts that are represented as smooth vertical lines that extend from the pleural line to the far field while obliterating A-lines [34, 35]. Particularly in COVID-19, B-lines are commonly visualized in the posterior-lateral lung zones in the early phases of the disease [33]. In addition to pleuropathies, consolidations are pathological and can be identified peripherally and inferior to or disrupting the pleural line [9, 18, 30, 31]. Air bronchograms are visualized as bright opacities distal to a
Table 1 Modified PoCUS machine disinfection checklist: COVID-19 point-of-view

Minimize the amount of additional equipment on the ultrasound cart and if pos-
sible designate certain devices as COVID-19-specific
If possible, cover the machine and probe in a protective plastic sheath
Use single-use gel packets rather than a reusable bottle
Inspect the probe and thoroughly sanitize the machine/reusable plastic sheath
using approved-disinfectant wipes while wearing personal protective equipment
(PPE)
Remove the machine from the patient's room followed by doffing PPE
Reinspect the machine; re-apply new gloves and disinfect the machine once again

${ }^{\text {a }}$ Disinfecting hand-held PoCUS device can be less strenuous with the use of smaller disposable plastic sheaths 
consolidation when the ultrasound wave reflects off of an air-filled bronchus $[9,18,31]$. As previously stated, pleural effusions are uncommon in COVID-19 but can be seen clearly as anechoic regions found cephalad to the diaphragm $[9,18,30,31]$.

\section{PoCUS for COVID-19 cardiac involvement}

PoCUS has played a pivotal role in monitoring patients' cardiac function, especially those with pre-existing cardiovascular co-morbidities $[10,12]$. A point-of-care echocardiogram may be useful to evaluate patient status when they become hemodynamically unstable or have elevated cardiac biomarkers [12]. Cardiac involvement in COVID-19 presents in a large proportion of patients and as a multitude of pathologies. Cardiac involvement of the disease is very common amongst patients and the process can be either via primary viral infection or secondary activation of the immune cascade $[14,23]$. These processes directly affect cardiac function from resultant myocarditis and pulmonary embolisms (PE) [17, 23].

PoCUS has high accuracy when used to evaluate left and right ventricular function, valvular dysfunction, pericardial effusion and to calculate stroke volume [10, 36]. In monitoring PE occurrence, PoCUS has been used to assess right ventricular function and resulting pulmonary hypertension. The ability to identify these findings using cardiac PoCUS provides information for whether or not additional management is required [37].

\section{PoCUS for COVID-19 vascular involvement}

Fluid status changes and vascular abnormalities are commonly associated with COVID-19. It has been suggested that inflammation caused by COVID-19 may increase vascular permeability, thus reducing intravascular volume [38]. The hepatic, portal and intra-renal veins can be visualized using PoCUS to accurately assess fluid status in hospitalized COVID-19 patients [36]. If PoCUS is conducted serially, it is extremely beneficial in monitoring the effect of fluid administration in hemodynamically unstable patients [14].

Similarly, to other critically ill patients, COVID-19 has been associated with an increased risk for thromboembolic events [11]. A two-point compression PoCUS ultrasound examination may be utilized to assess for deep vein thrombosis (DVT) in the femoral and popliteal veins $[11,36]$. Given the difficulties in diagnosing a PE with superimposed lung involvement using CT pulmonary angiography, venous doppler studies are essential in guiding appropriate management [14].

Ultrasound-guided venous access provides physicians with an abundance of advantages. PoCUS can be utilized to assess for vessel thrombi prior to intravenous puncture, it may also help distinguish a vein from the artery [12]. It can also assess lung sliding pre- and post-procedural during central line placement to diagnose iatrogenic pneumothorax [12]. Particularly in COVID-19 patients, these parameters are very important to account for to prevent precipitating a thromboembolic event or worsening of concomitant lung involvement.

\section{PoCUS for COVID-19 in pregnancy}

PoCUS is particularly useful in pregnant women and monitoring disease progression of COVID-19 [39-42]. Accessibility at the bedside and elimination of radiation exposure enables ultrasound to be used to serially track COVID-19 manifestations while pregnant [40]. Although CT imaging is used to diagnose and monitor pulmonary disease progression in many COVID-19 patients, the downsides of radiation exposure outweigh the benefits. In theory, reducing the amount of radiation used would be the solution, however, CT with reduced radiation levels has been found to be insensitive in the early and/or mild COVID-19 cases [43].LUS is proven to correlate with $\mathrm{CT}$ images and is therefore feasible during pregnancy $[3,44]$.

In addition, obstetricians and gynecologists may be adequately trained to perform a fetal ultrasound followed by a LUS examination in pregnant patients with a suspected or known COVID-19 diagnosis [45, 46]. This minimizes the need for various ultrasound operators and equipment, reducing the exposure to potential infection. Moreover, the severity of the disease seen on LUS may influence treatment decisions and subsequently the outcome of the pregnancy [43].

\section{PoCUS for COVID-19 in neonates and children}

Ultrasonography is a safe and effective tool to monitor neonatal lung and cardiac diseases. Its neonatal application in COVID-19 has been used to assess the progression of lung involvement [47-50]. Interestingly, some neonates with COVID-19 were found to have signs of lung involvement on LUS, that did not coincide with any respiratory decompensation, commonly seen in adults [51]. Similarly to adults, B-lines, pleural irregularities, and consolidations were noted in neonates [51].

Although much of the discussion around COVID-19 and ultrasound refers to its use in adults, it is also a practical tool when it comes to assessing infection in children. A majority of children are asymptomatic, yet those who present with symptoms commonly have a fever, cough or both [52]. It has been suggested that in early disease, not all children display characteristic COVID-19 LUS findings [53]. However, when they do, ultrasound findings include pleural irregularities, sub-pleural consolidations and B lines [54]. Therefore, in 


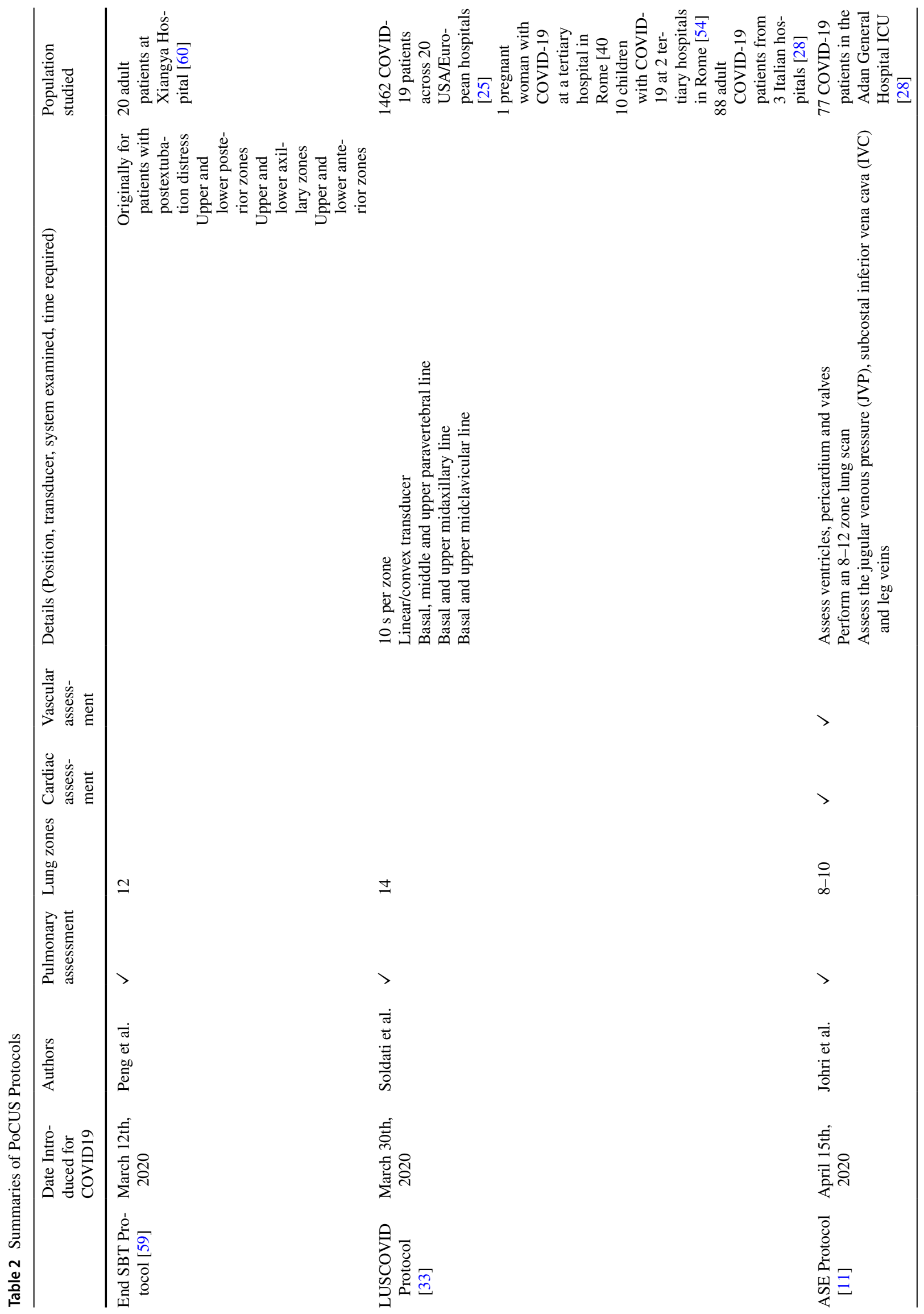


Journal of Ultrasound (2022) 25:207-215

211

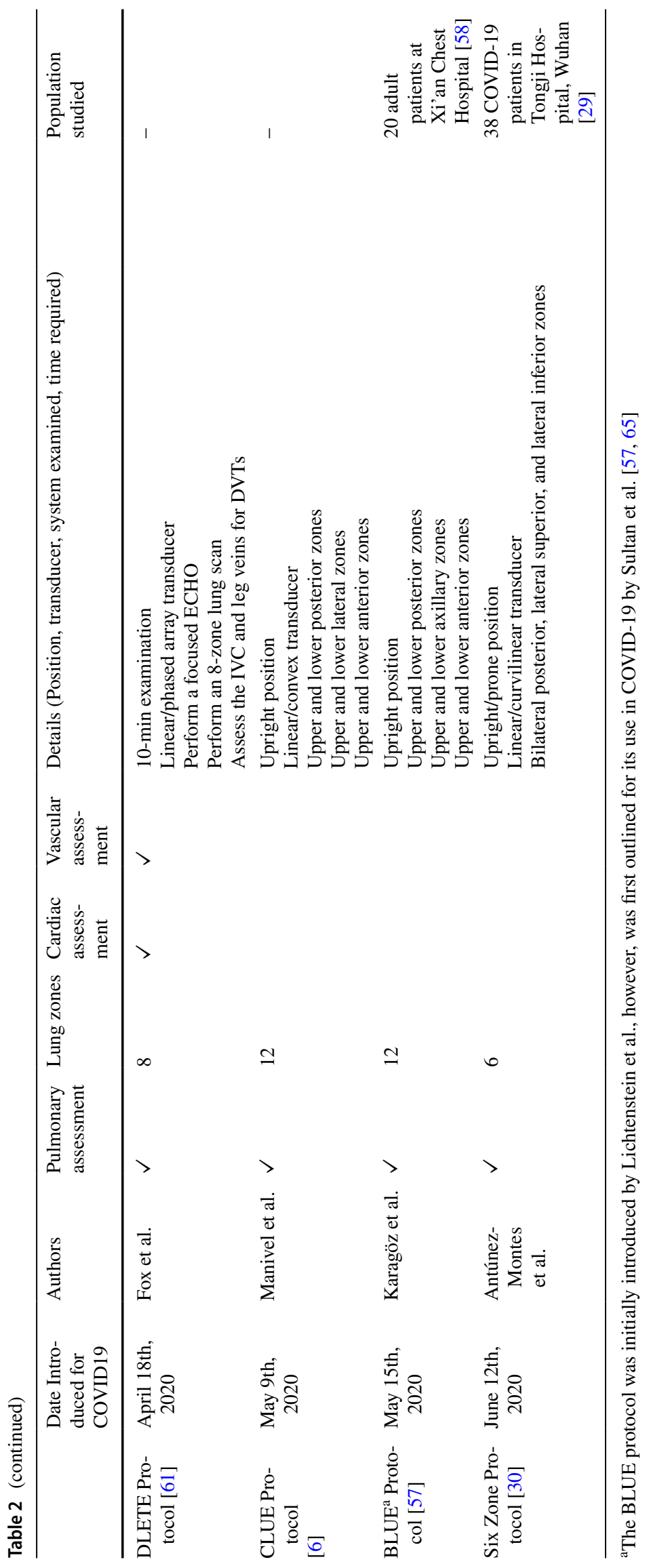

Springer 
Fig. 1. 12 zone scanning protocol showing 2 anterior zones, 2 lateral zones and 2 posterior zones. Asterisks (*) indicate the posterobasal areas where COVID19 changes occur most frequently

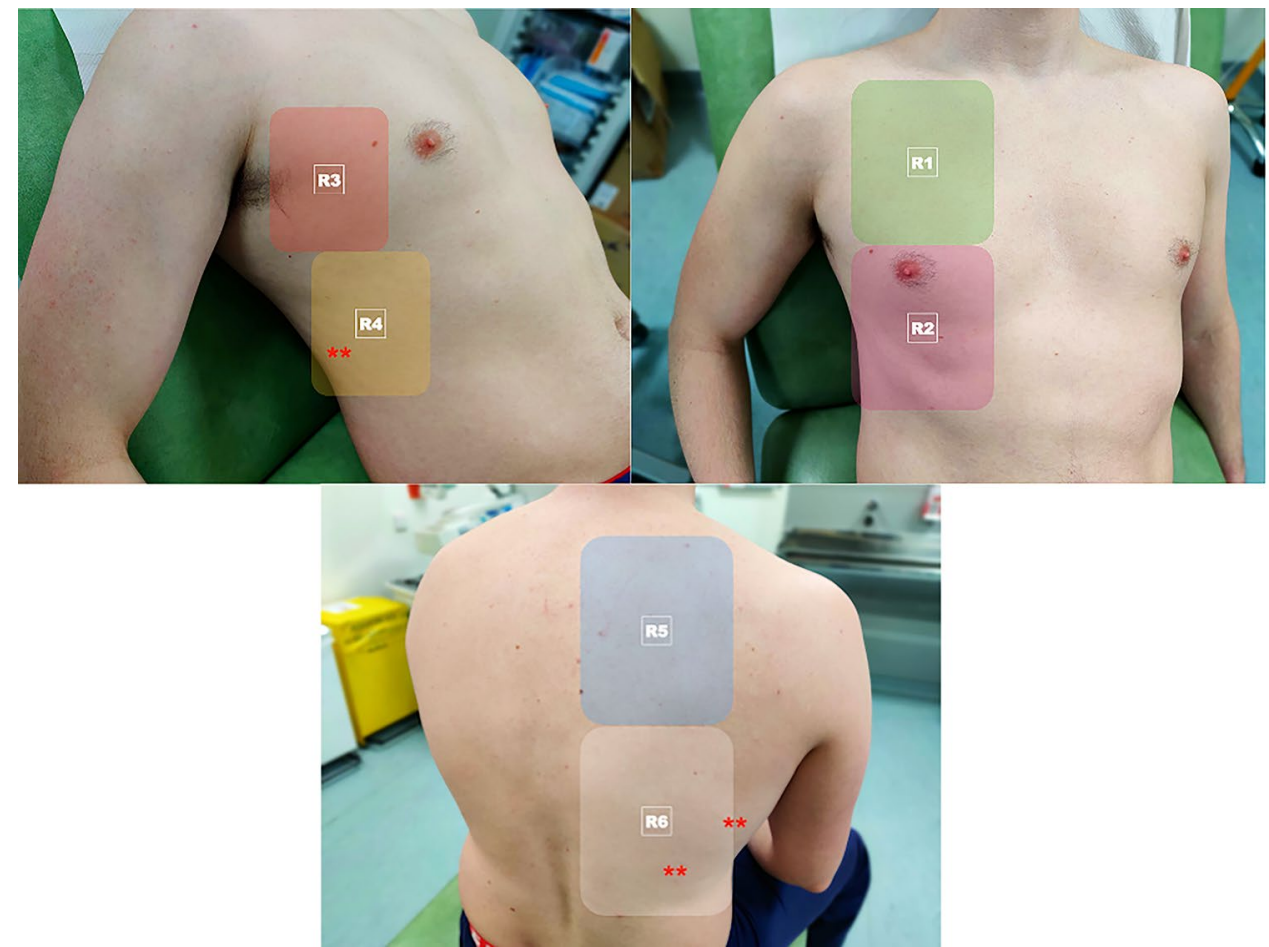

addition to the use of LUS in other viral pneumonias, there is compelling evidence suggesting LUS should be used at the bedside in children [54].

Less frequently, children have been reported to develop multi-system inflammatory syndrome (MIS); a syndrome which closely mimics Kawasaki disease [55]. This can present as a rash, bilateral conjunctivitis, myocardial dysfunction, shock, coagulopathy or acute gastrointestinal distress [55]. A large majority of these children with MIS present with cardiac manifestations including arrhythmias, ventricular dysfunction and coronary dilatation, primarily caused by focal necrosis and fibrosis of the myocardium [33]. Furthermore, to prevent the overuse of CT on children, ultrasound may be a useful modality in the evaluation of cardiac dysfunction, fluid status and vascular abnormalities in the COVID-19 pediatric population [54-56].

\section{PoCUS scanning protocol options in COVID-19}

As a result of COVID-19, numerous PoCUS scanning protocols have been introduced to best assess patient status. (Table 2) Each protocol was designed to quickly assess COVID-19 patients in the emergency department or the intensive care unit. This not only minimizes exposure to potential respiratory droplets but also gives the physicians a more well-rounded clinical picture of the patient's current condition.

The 'under 2-min' Six Zone LUS Protocol [29, 30] proposes that the operator situates themselves posterior to the patient sitting upright, and three scans are conducted bilaterally: posterior zone of the chest wall, lateral superior and inferior zones of the axilla (Fig. 1). The CLUE Protocol [6], BLUE Protocol $[57,58]$ and the end spontaneous breathing trial (SBT) Protocol $[59,60]$ involve a more comprehensive twelve-zone LUS assessment. The CLUE Protocol produces a score of 0-3 per zone, with 3 representing more severe disease. The BLUE Protocol offers insight into what the underlying pulmonary pathology may be depending on the specific LUS findings observed. In regard to COVID-19, the presence of multiple diffuse B-lines indicates pulmonary edema, whereas consolidations indicate pneumonia [57]. The end SBT LUS protocol provides a score of 0 for a normal LUS, 1 or 2 for the presence of B-lines and 3 for consolidation [59]. The LUSCOVID Protocol [25, 28, 33, $39,54,61,62]$ assesses 14-zones thus it is the most extensive LUS protocol.

The ASE Protocol [11, 63] and DLETE [64] are the most comprehensive and are comprised of cardiac, lung and venous assessment which encompasses the main manifestations of COVID-19.

\section{Conclusion}

PoCUS' usefulness as an adjunctive tool in the management of COVID-19 is strong. The portability, availability and the real-time nature of ultrasound provides physicians with a 
safe imaging modality for pulmonary, cardiac and vascular COVID-19 manifestations. With no risk of radiation exposure, ultrasonography is beneficial when used to assess lung involvement in pregnant women. Additionally, ultrasound is practical and effective for use in pediatric COVID-19 patients.

Funding Open Access funding provided by the IReL Consortium. No funding was received during the creation of this review article.

\section{Declarations}

Competing interests There are no conflicts of interest.

Ethical approval If human beings have participated in the research (**) All procedures performed in studies involving human participants were in accordance with the ethical standards of the institutional and/ or national research committee and with the 1964 Helsinki declaration and its later amendments or comparable ethical standards. If human beings have NOT participated in the research. This article does not contain any studies with human participants or animals performed by any of the authors.

Informed consent Informed consent was obtained from all individual participants included in the study.

Open Access This article is licensed under a Creative Commons Attribution 4.0 International License, which permits use, sharing, adaptation, distribution and reproduction in any medium or format, as long as you give appropriate credit to the original author(s) and the source, provide a link to the Creative Commons licence, and indicate if changes were made. The images or other third party material in this article are included in the article's Creative Commons licence, unless indicated otherwise in a credit line to the material. If material is not included in the article's Creative Commons licence and your intended use is not permitted by statutory regulation or exceeds the permitted use, you will need to obtain permission directly from the copyright holder. To view a copy of this licence, visit http://creativecommons.org/licenses/by/4.0/.

\section{References}

1. Guan W, Ni Z, Hu Y, Liang W, Ou C, He J et al (2020) Clinical Characteristics of coronavirus disease 2019 in China. N Engl J Med 382(18):1708-1720

2. Li Q, Guan X, Wu P, Wang X, Zhou L, Tong Y et al (2020) Early Transmission dynamics in Wuhan, China, of novel coronavirusinfected pneumonia. N Engl J Med 382(13):1199-1207

3. Meyerowitz E, Richterman A, Gandhi R, Sax P (2020) Transmission of SARS-CoV-2: a review of viral, host, and environmental factors. Annals Int Med. 21:771

4. Stokes E, Zambrano L, Anderson K, Marder E, Raz K, El Burai FS et al (2020) Coronavirus disease 2019 case surveillanceUnited States, January 22-May 30, 2020. MMWR Morb Mortal Wkly Rep 69(24):759-765

5. Timeline: WHO's COVID-19 response [Internet]. Who.int. 2020. https://www.who.int/emergencies/diseases/novel-coronavirus2019/interactive-timeline\#event-0. Accessed 24 Sept 2020
6. Manivel V, Lesnewski A, Shamim S, Carbonatto G, Govindan T (2020) CLUE: COVID-19 lung ultrasound in emergency department. Emerg Med Australas 32(4):694-696

7. Rafiee M, Parsaei F, RahimiPordanjani S, Amiri V, Sabour S (2020) A review on applicable and available paraclinical methods for diagnosis of coronavirus disease-19. Arch Iran Med 23(11):794-800

8. Pare J, Camelo I, Mayo K, Leo M, Dugas J, Nelson K et al (2020) Point-of-care lung ultrasound is more sensitive than chest radiograph for evaluation of COVID-19. Western J Emerg Med. 21(4):771

9. Moore S, Gardiner E (2020) Point of care and intensive care lung ultrasound: a reference guide for practitioners during COVID-19. Radiography 26(4):e297-e302

10. Khanji M, Ricci F, Patel R, Chahal A, Bhattacharyya S, Galusko $\mathrm{V}$ et al (2020) The role of hand-held ultrasound for cardiopulmonary assessment during a pandemic. Progress Cardiovasc Dis. 63:690-695

11. Johri A, Galen B, Kirkpatrick J, Lanspa M, Mulvagh S, Thamman R (2020) ASE statement on point-of-care ultrasound during the 2019 novel coronavirus pandemic. J Am SocEchocardiogr 33(6):670-673

12. Mathews B, Koenig S, Kurian L, Galen B, Mints G, Liu G et al (2020) Clinical progress note: point-of-care ultrasound applications in COVID-19. J Hosp Med 15(6):353-355

13. Rubulotta F, Soliman-Aboumarie H, Filbey K, Geldner G, Kuck K, Ganau M et al (2020) Technologies to optimize the care of severe COVID-19 patients for health care providers challenged by limited resources. AnesthAnalg 131(2):351-364

14. Chiem A, Shibata J, Lim G, Liu Y. Pick Up Your Probes. J Ultrasound Med. 2020.

15. Kim D, Jelic T, Woo M, Heslop C, Olszynski P (2020) Just the Facts: Recommendations on point-of-care ultrasound use and machine infection control during the coronavirus disease 2019 pandemic. CJEM 22(4):445-449

16. Gogna A, Yogendra P, Lee S, Aziz A, Cheong E, Chan L et al (2020) Diagnostic ultrasound services during the coronavirus disease (COVID-19) pandemic. Am J Roentgenol. 215:1-6

17. Abrams E, Rose G, Fields J, Esener D (2020) Point-of-Care ultrasound in the evaluation of COVID-19. J Emerg Med. 59:403-408

18. McDermott C, Daly J, Carley S (2020) Combatting COVID-19: is ultrasound an important piece in the diagnostic puzzle? Emerg Med J 37(10):644-649

19. Smallwood N, Walden A, Parulekar P, Dachsel M (2020) Should point-of-care ultrasound become part of healthcare worker testing for COVID? Clin Med 20(5):486-487

20. Tierney D, Huelster J, Overgaard J, Plunkett M, Boland L, St. Hill C et al (2020) Comparative performance of pulmonary ultrasound, chest radiograph, and $\mathrm{CT}$ among patients with acute respiratory failure*. Crit Care Med. 48(2):151-157

21. Yang Y, Huang Y, Gao F, Yuan L, Wang Z (2020) Lung ultrasonography versus chest CT in COVID-19 pneumonia: a twocentered retrospective comparison study from China. Intensive Care Med 46(9):1761-1763

22. Huang G, Vengerovsky A, Morris A, Town J, Carlbom D, Kwon Y (2020) Development of a COVID-19 point-of-care ultrasound protocol. J Am SocEchocardiogr 33(7):903-905

23. Agricola E, Beneduce A, Esposito A, Ingallina G, Palumbo D, Palmisano A et al (2020) Heart and lung multimodality imaging in COVID-19. JACC Cardiovascular Imag. 13(8):1792-1808

24. Xu J, Ma X, Bai L, Wang M, Deng W, Ning N (2020) A systematic review of etiology, epidemiology, clinical manifestations, image findings, and medication of 2019 Corona Virus Disease-19 in Wuhan, China. Medicine 99(42):e22688

25. Volpicelli G, Gargani L, Perlini S, Spinelli S, Barbieri G, Lanotte A et al (2021) Lung ultrasound for the early diagnosis of 
COVID-19 pneumonia: an international multicenter study. Intens Care Med. https://doi.org/10.1093/ehjci/jeaa163

26. Duggan N, Shokoohi H, Liteplo A, Huang C, Goldsmith A (2020) Best practice recommendations for point-of-care lung ultrasound in patients with suspected COVID-19. J Emerg Med. 59:515-520

27. Smargiassi A, Soldati G, Torri E, Mento F, Milardi D, Giacomo $P$ et al (2020) Lung ultrasound for COVID-19 patchy pneumonia: extended or limited evaluations? J Ultrasound Med. 40:521-528

28. Mento F, Perrone T, Macioce V, Tursi F, Buonsenso D, Torri E et al (2020) On the impact of different lung ultrasound imaging protocols in the evaluation of patients affected by coronavirus disease 2019. J Ultrasound Med. https://doi.org/10.1002/jum. 15580

29. Zhu F, Zhao X, Wang T, Wang Z, Guo F, Xue H et al (2020) Ultrasonic characteristics and severity assessment of lung ultrasound in COVID-19 pneumonia in Wuhan, China: a retrospective Observational Study. Engineering. https://doi.org/10. 1016/j.eng.2020.09.007

30. Antúnez-Montes O, Buonsenso D (2020) Routine use of pointof-care lung ultrasound during the COVID-19 pandemic. Med Intensiva. https://doi.org/10.1016/j.medin.2020.04.010

31. Smith M, Hayward S, Innes S, Miller A (2020) Point-of-care lung ultrasound in patients with COVID-19-a narrative review. Anaesthesia 75(8):1096-1104

32. Smargiassi A, Soldati G, Borghetti A, Scoppettuolo G, Tamburrini E, Testa A et al (2020) Lung ultrasonography for early management of patients with respiratory symptoms during COVID-19 pandemic. J Ultrasound 23(4):449-456

33. Soldati G, Smargiassi A, Inchingolo R, Buonsenso D, Perrone T, Briganti D et al (2020) Proposal for international standardization of the use of lung ultrasound for patients with COVID -19. J Ultrasound Med 39(7):1413-1419

34. Soldati G, Demi M, Inchingolo R, Smargiassi A, Demi L (2016) On the physical basis of pulmonary sonographic interstitial syndrome. J Ultrasound Med 35(10):2075-2086

35. Demi L, Demi M, Smargiassi A, Inchingolo R, Faita F, Soldati G (2014) Ultrasonography in lung pathologies: new perspectives. MultidisciplinRespirat Med 9(1):27

36. Koratala A, Ronco C, Kazory A (2020) Need for objective assessment of volume status in critically Ill patients with COVID-19: The Tri-POCUS approach. Cardiorenal Med 10(4):209-216

37. Zhang L, Wang B, Zhou J, Kirkpatrick J, Xie M, Johri A (2020) Bedside focused cardiac ultrasound in COVID-19 from the Wuhan Epicenter: The role of cardiac point-of-care ultrasound, limited transthoracic echocardiography, and critical care echocardiography. J Am SocEchocardiogr 33(6):676-682

38. Thalappillil R, White R, Tam C (2020) POCUS to guide fluid therapy in COVID-19. J CardiothoracVascAnesth 34(10):2854-2856

39. Inchingolo R, Smargiassi A, Moro F, Buonsenso D, Salvi S, Del Giacomo P et al (2020) The diagnosis of pneumonia in a pregnant woman with coronavirus disease 2019 using maternal lung ultrasound. Am J ObstetGynecol 223(1):9-11

40. Buonsenso D, Raffaelli F, Tamburrini E, Biasucci D, Salvi S, Smargiassi A et al (2020) Clinical role of lung ultrasound for diagnosis and monitoring of COVID-19 pneumonia in pregnant women. Ultrasound ObstetGynecol 56(1):106-109

41. Yassa M, Mutlu M, Kalafat E, Birol P, Yirmibeş C, Tekin A et al (2020) How to perform and interpret the lung ultrasound by the obstetricians in pregnant women during the SARS-CoV-2 pandemic. J Turkish Soc Obstetric Gynecol 17(3):225-232

42. Yassa M, Mutlu M, Birol P, Kuzan T, Kalafat E, Usta C et al (2020) Lung ultrasonography in pregnant women during the COVID-19 pandemic: an interobserver agreement study among obstetricians. Ultrasonography 39(4):340-349
43. Yassa M, Birol P, Mutlu A, Tekin A, Sandal K, Tug N (2020) Lung Ultrasound can influence the clinical treatment of pregnant women with COVID -19. J Ultrasound Med. 40(1):191-203

44. Kalafat E, Yaprak E, Cinar G, Varli B, Ozisik S, Uzun C et al (2020) Lung ultrasound and computed tomographic findings in pregnant woman with COVID-19. Ultrasound ObstetGynecol 55(6):835-837

45. Moro F, Buonsenso D, Merwe J, Bourne T, Deprest J, Froyman $\mathrm{W}$ et al. A Prospective international lung ultrasound analysis study in tertiary maternity wards during the severe acute respiratory syndrome coronavirus 2 pandemic. J Ultrasound Med. 2020.

46. Buonsenso D, Moro F, Inchingolo R, Smargiassi A, Demi L, Soldati G et al (2020) Effectiveness of rapid lung ultrasound training program for gynecologists and obstetricians managing pregnant women with suspected COVID-19. Ultrasound ObstetGynecol 56(1):110-111

47. Tsankov B, Allaire J, Irvine M, Lopez A, Sauvé L, Vallance B et al (2020) Severe COVID-19 infection and pediatric comorbidities: a systematic review and meta-analysis. Int J Infect Dis. 103:246-256

48. Zhang C, Gu J, Chen Q, Deng N, Li J, Huang L et al (2020) Clinical and epidemiological characteristics of pediatric SARSCoV-2 infections in China: a multicenter case series. PLoS Med 17(6): 1003130

49. Guo C, He L, Yin J, Meng X, Tan W, Yang G et al (2020) Epidemiological and clinical features of pediatric COVID-19. BMC Med. 18(1):1-7

50. Parri N, Lenge M, Cantoni B, Arrighini A, Romanengo M, Urbino A et al (2020) COVID-19 in 17 Italian Pediatric Emergency Departments. Pediatrics 146:e20201235

51. Gregorio-Hernández R, Escobar-Izquierdo A, Cobas-Pazos J, Martínez-Gimeno A (2020) Point-of-care lung ultrasound in three neonates with COVID-19. Eur J Pediatr 179(8):1279-1285

52. Wang Z, Zhou Q, Wang C, Shi Q, Lu S, Ma Y et al (2020) Clinical characteristics of children with COVID-19: a rapid review and meta-analysis. Annals Translat Med 8(10):620-620

53. Scheier E, Guri A, Balla U (2020) Lung ultrasound cannot be used to screen for Covid-19 in children. Eur Rev PharmacolSci 9:4623-4624

54. Musolino A, Supino M, Buonsenso D, Ferro V, Valentini P, Magistrelli A et al (2020) Lung ultrasound in children with COVID-19: PREliminary findings. Ultrasound Med Biol 46(8):2094-2098

55. Jiang L, Tang K, Levin M, Irfan O, Morris S, Wilson K et al (2020) COVID-19 and multisystem inflammatory syndrome in children and adolescents. Lancet Infect Dis. 20(11):e276-e288

56. Liu E, Smyth R, Luo Z, Qaseem A, Mathew J, Lu Q et al (2020) Rapid advice guidelines for management of children with COVID-19. Annals Translat Med 8(10):617-617

57. Karagöz A, Sağlam C, Demirbaş H, Korkut S, Ünlüer E (2020) Accuracy of bedside lung ultrasound as a rapid triage tool for suspected Covid-19 Cases. Ultrasound Q 36(4):339-344

58. Huang Y, Wang S, Liu Y, Zhang Y, Zheng C, Zheng Y et al (2020) A Preliminary study on the ultrasonic manifestations of peripulmonary lesions of non-critical novel coronavirus pneumonia (COVID-19). SSRN Electron J. 46:2908-2917

59. Soummer A, Perbet S, Brisson H, Arbelot C, Constantin J, Lu Q et al (2012) Ultrasound assessment of lung aeration loss during a successful weaning trial predicts postextubation distress*. Crit Care Med 40(7):2064-2072

60. Peng Q, Wang X, Zhang L (2020) Findings of lung ultrasonography of novel corona virus pneumonia during the 2019-2020 epidemic. Intensive Care Med 46(5):849-850

61. Perrone T, Soldati G, Padovini L, Fiengo A, Lettieri G, Sabatini $\mathrm{U}$ et al (2020) A new lung ultrasound protocol able to predict 
worsening in patients affected by severe acute respiratory syndrome coronavirus 2 pneumonia. J Ultrasound Med. https://doi. org/10.1002/jum. 15548

62. Smargiassi A, Soldati G, Torri E, Mento F, Milardi D, Del Giacomo P et al (2020) Lung ultrasound for COVID -19 patchy pneumonia. J Ultrasound Med 40(3):521-528

63. Bitar Z, Shamsah M, Bamasood O, Maadarani O, Alfoudri H (2021) Point-of-Care ultrasound for COVID-19 pneumonia patients in the ICU. J CardiovascImag 29(1):60

64. Fox S, Dugar S (2020) Point-of-care ultrasound and COVID-19. Cleveland Clin J Med. https://doi.org/10.3949/ccjm.87a.ccc019
65. Lichtenstein D (2014) Lung ultrasound in the critically ill. Ann Intensive Care 4(1):1

Publisher's Note Springer Nature remains neutral with regard to jurisdictional claims in published maps and institutional affiliations. 